\title{
Outcomes and relapse patterns following chemotherapy in advanced Hodgkin lymphoma in the positron emission tomography era
}

This article was published in the following Dove Press journal: Blood and Lymphatic Cancer:Targets and Therapy

\author{
Carminia Lapuz ${ }^{1-3}$ \\ Anoop K Enjeti ${ }^{2,4}$ \\ Peter C O'Brien ${ }^{1,2}$ \\ Anne L Capp ${ }^{1,2}$ \\ Elizabeth G Holliday ${ }^{2,5}$ \\ Sanjiv A Gupta ${ }^{1,2}$ \\ 'Department of Radiation Oncology, \\ Calvary Mater Newcastle, Newcastle, \\ NSW, Australia; ${ }^{2}$ Faculty of Medicine \\ and Public Health, University \\ of Newcastle, Newcastle, NSW, \\ Australia; ${ }^{3}$ Department of Radiation \\ Oncology, Olivia Newton-John Cancer \\ Centre, Austin Health, VIC, Australia; \\ ${ }^{4}$ Department of Haematology, Calvary \\ Mater Newcastle, Newcastle, NSW, \\ Australia; ${ }^{5}$ Faculty of Public Health, \\ Hunter Medical Research Institute, \\ Newcastle, NSW, Australia
}

Background: This study evaluated relapse patterns and survival in advanced Hodgkin lymphoma (HL) patients treated with doxorubicin, bleomycin, vinblastine and dacarbazine (ABVD) with positron emission tomography (PET) used for staging and response assessment.

Patients and methods: Patients aged 18 years or above with newly diagnosed histologically proven Stage III or IV HL treated with ABVD at Calvary Mater Newcastle from January 2005 to December 2012 were included in this study. All patients underwent pre-chemotherapy staging with ${ }^{18} \mathrm{~F}$-fluorodeoxyglucose PET or PET/computed tomography and post-chemotherapy PET or PET/computed tomography for the assessment of response.

Results: Forty-three patients were included in the study. The 5-year disease-free survival, progression-free survival and overall survival were $88 \%, 74 \%$ and $86 \%$, respectively. PET complete response was seen in 35 patients $(81 \%)$, and the 5-year overall survival for this group was $94 \%$. Relapse following a PET complete response was low (three patients) and occurred predominantly at the initial sites of disease. Four of five patients with bulky disease received consolidative radiotherapy and no in-field relapses were observed.

Conclusion: Advanced stage HL with a PET complete response following ABVD is associated with an excellent prognosis.

Keywords: Hodgkin lymphoma, advanced, PET scan

\section{Introduction}

Combination chemotherapy with doxorubicin, bleomycin, vinblastine and dacarbazine (ABVD) is a standard regimen used in advanced Hodgkin lymphoma (HL), resulting in failure-free survival rates of $60 \%-70 \%$ and overall survival rates of $80 \%-90 \%{ }^{1-7}$ Despite the excellent overall survival, $\sim 30 \%-40 \%$ of patients relapse within the first 2 years after ABVD and require salvage treatment. ${ }^{2,4,8}$

Published patterns of relapse studies in advanced HL suggest most relapses occur at the sites of initial disease. ${ }^{9,10}$ Consequently, some advocate the use of consolidative radiotherapy (RT) to minimize the risk of relapse. However, these patterns of relapse studies used chemotherapy regimens that vary from current practice. Furthermore, these studies did not routinely use positron emission tomography (PET) to assess response to chemotherapy. PET offers additional prognostic information and its use has become the standard for initial staging and for the assessment of response after chemotherapy in HL. ${ }^{11}$

RT, if used, is generally done so in the setting of bulky disease at presentation or if there is a partial response following chemotherapy. ${ }^{12,13}$ The definition of bulky is varied,
Correspondence: Sanjiv A Gupta Department of Radiation Oncology, Calvary Mater Newcastle, Hunter Region Mail Centre, Newcastle, NSW 23I0, Australia

Tel +6I 240143640

Fax +6I 240143169

Email Sanjiv.gupta@calvarymater.org.au 
but more recently, there has been a recommendation to use $10 \mathrm{~cm}$ as the cut-off, with measurements taken in the axial, coronal and sagittal planes. ${ }^{14}$ Prospective and retrospective studies examining the role of consolidative RT in advanced HL have been mixed in results, with some showing a benefit in certain patient groups ${ }^{15,17}$ and others showing no benefit. ${ }^{18}$

The aim of this study is to assess the patterns of relapse and outcomes in PET-staged advanced stage HL treated with ABVD chemotherapy and assessed with post-chemotherapy PET scan, in order to help guide selection for more intensive therapy such as the addition of consolidative RT.

\section{Patients and methods}

Adult patients aged over 18 years and with newly diagnosed histologically proven Stage III or IV HL treated with ABVD chemotherapy at Calvary Mater Newcastle, New South Wales, Australia from January 2005 to December 2012 were included in this retrospective study. All patients underwent pre-chemotherapy staging with ${ }^{18} \mathrm{~F}$-fluorodeoxyglucose PET or PET/computed tomography (CT) and post-chemotherapy PET or PET/CT for assessment of response. This study was approved by the ethics committee of Calvary Mater Newcastle. Patient consent was not required as the study was a retrospective audit and was deemed low risk. Patient confidentiality was maintained using de-identified data.

Information was collected on patient demographics, disease characteristics at diagnosis (including international prognostic score, ${ }^{19}$ the presence of bulky disease [defined as any single nodal mass $\geq 10 \mathrm{~cm}$ in diameter]), treatment received (number of cycles of ABVD and RT details), PET response following chemotherapy and sites of disease recurrence.

Patients were treated with six to eight cycles of ABVD (doxorubicin $25 \mathrm{mg} / \mathrm{m}^{2}$, vinblastine $6 \mathrm{mg} / \mathrm{m}^{2}$, bleomycin 10,000 international units $/ \mathrm{m}^{2}$ and dacarbazine $\left.375 \mathrm{mg} / \mathrm{m}^{2}\right) .{ }^{1}$ Patients who received more intensive therapy with bleomycin, etoposide, doxorubicin, cyclophosphamide, vincristine, procarbazine and prednisone (BEACOPP) were excluded from this study.

Interim PET scans during chemotherapy were performed at the discretion of the treating hematologist or medical oncologist. The post-chemotherapy PET scan was used to confirm response and was performed within 3 months of ABVD completion in $98 \%$ of patients. Definitions of response were based on the response criteria for malignant lymphoma. ${ }^{20}$

Patients were given consolidative RT at the discretion of treating clinicians. Involved field RT was administered using anterior and posterior fields with 6-18 MV photons from a linear accelerator. RT dose was 30-36 Gy in 15-18 fractions given over 3-4 weeks.
Patients were subsequently evaluated 3-6 monthly in the first year after treatment and 6-12 monthly thereafter. If clinical features were suggestive of recurrence, then imaging was performed at the discretion of the treating clinician.

\section{Statistical analysis}

Patterns of relapse were assessed relative to sites of initial disease and RT field if given. Disease-free survival (DFS), progression-free survival (PFS) and overall survival (OS) were assessed using the Kaplan-Meier survival estimator. DFS was defined as the time from attainment of complete response (CR), as assessed on post-chemotherapy PET, to relapse or death. PFS was defined as the time from the first day of chemotherapy until progression or death. OS was defined as the time from the first day of chemotherapy to death from any cause. The Cox proportional hazards regression method was used to identify prognostic factors for DFS, PFS and OS. Due to the small number of patients, only bulky disease, presence of B symptoms, stage and age were examined. All statistical analyses were programmed using Stata v13.0 (StataCorp LP, College Station, TX, USA).

\section{Results}

\section{Baseline characteristics}

A total of 43 patients were included in this study. Baseline patient characteristics are summarized in Table 1 . The median age at diagnosis was 43.0 years (range $18.5-74.9$ years); $58.1 \%$ were male and $41.9 \%$ were female. The most common histologic subtype was nodular sclerosis $(44.2 \%)$ followed by mixed cellularity $(30.2 \%)$. Stage III disease was seen in $62.8 \%$ of patients, compared with Stage IV disease in $37.2 \%$ of patients. Five patients $(11.6 \%)$ had bulky disease at presentation and 28 patients $(65.1 \%)$ had B symptoms. Median number of sites involved at presentation was 7 (range 2-14). Extranodal disease was seen in 17 patients (39.5\%) and splenic involvement was seen in 21 patients $(48.8 \%)$. Most patients had an international prognostic score of 2 or $3(58.2 \%)$.

The majority of patients $(74.4 \%)$ received six cycles of ABVD with a range of two to eight cycles. Of the five patients who received less than six cycles, four patients ceased ABVD due to toxicity and one patient was found to have progression on interim PET and was given second-line chemotherapy.

\section{Patterns of relapse/progression}

The median follow-up time was 45.7 months (range 6.6-102.1 months). PET CR after ABVD was seen in 35 patients $(81.4 \%)$, partial response $(\mathrm{PR})$ in 3 patients $(7.0 \%)$ and progression in 5 patients $(11.6 \%)$. Of the patients with 
Table I Baseline patient characteristics

\begin{tabular}{ll}
\hline Characteristics & Total (\%) \\
\hline Age, median (range) & 43.0 years (18.5-74.9) \\
Sex & \\
Male & $25(58.1)$ \\
Female & $18(41.9)$ \\
Histologic subtype & \\
Nodular sclerosis & $19(44.2)$ \\
Mixed cellularity & $13(30.2)$ \\
Lymphocyte depleted & $3(7.0)$ \\
Lymphocyte rich & $2(4.7)$ \\
Nodular lymphocyte predominant & $2(4.7)$ \\
Classical Hodgkin lymphoma, type not & $4(9.3)$ \\
specified & \\
Stage & \\
III & $27(63)$ \\
IV & $16(37)$ \\
Bulky disease & \\
Yes & $5(12)$ \\
No & $38(88)$ \\
B symptoms & \\
Yes & $28(65)$ \\
No & $15(35)$ \\
Number of sites, median (range) & $7(2-14)$ \\
Extranodal sites & $17(39.5)$ \\
Yes & $26(60.5)$ \\
No & $21(48.8)$ \\
Splenic involvement & $22(51.2)$ \\
Yes & $6(14)$ \\
No & $25(58)$ \\
International prognostic score & $12(28)$ \\
$0-I$ & \\
$2-3$ & \\
$4-7$ & \\
\hline & \\
&
\end{tabular}

PET CR, three patients (9\%) relapsed (Figure 1). Two of these patients relapsed within sites of initial disease only, which were all nodal and not initially bulky. One patient relapsed in both initial sites (which were nodal) and a new site of disease (which was a previously uninvolved bone). Relapses occurred within 2 years of attainment of PET CR. All patients were treated with second-line systemic therapy. One patient remains alive and disease free, one patient died of disease and one patient is alive with disease.

Of the three patients with a PET PR after completion of ABVD, two had a single site of residual disease and received consolidative RT. One of these patients is alive and disease free, while the other patient relapsed in new sites (multiple bone sites) during RT. She was successfully salvaged with chemotherapy and an autologous stem cell transplant and is alive without disease. The third patient with PET PR after ABVD had three sites of residual disease, but was not fit enough for salvage therapy and received best supportive care.

Of the five patients with progression on post-chemotherapy PET, one patient had progression in an initial site of disease only, while the other four patients progressed at an initial site of disease as well as developing new sites of disease (Figure 2). Salvage treatment consisted of high-dose chemotherapy with autologous stem cell transplant for three patients (with one patient also receiving RT), RT alone for one patient and palliative chemotherapy for one patient. Two patients are alive without disease, two patients are alive with disease and one patient died of disease.

\section{Interim PET scans}

Thirty patients (69.8\%) had interim PET scans, of whom 19 patients had a CR, 10 had a PR and 1 had progression. For 3 of the 10 patients with an interim PET PR, the number of ABVD cycles was increased from six to eight and all three had a postchemotherapy PET CR. For the other seven patients, there was no change in management based on the interim PET, with four having a subsequent post-chemotherapy PET CR and three $(30 \%)$ having progression on post-chemotherapy PET. The one patient with progression on the interim PET ceased ABVD and received salvage chemotherapy and RT. This patient remains alive with disease. One patient with an interim PET CR had six cycles of ABVD instead of the planned eight cycles and is alive without disease. Therefore, management was changed in five patients as a result of the interim PET scan.

\section{Disease-free survival, progression-free survival, overall survival}

The 5-year DFS was $88.0 \%$ (95\% CI: $71.1 \%-95.4 \%$; Figure 3). The 5-year PFS was $73.8 \%$ (95\% CI: $57.6 \%-$ $84.6 \%)$. The 5-year OS for the entire study group was $86.2 \%$ (95\% CI: $63.1 \%-95.3 \%$ ). The 5-year OS for patients with post-chemotherapy PET CR was $94.1 \%$ (95\% CI: 78.4\%-98.5\%; Figure 4). Four patients died. Two of these patients had a PET CR post-ABVD, and one death was due to progressive disease despite second-line systemic therapy (at 23.1 months) while the other was due to bleomycin-related lung toxicity (at 6.6 months). The other two patients died of progressive disease. One patient, who initially had a PET PR, was not fit for salvage therapy and died at 14.6 months. The other patient, who had progression on the post-ABVD PET scan, had multiple relapses despite salvage systemic therapy and died at 64.3 months. 


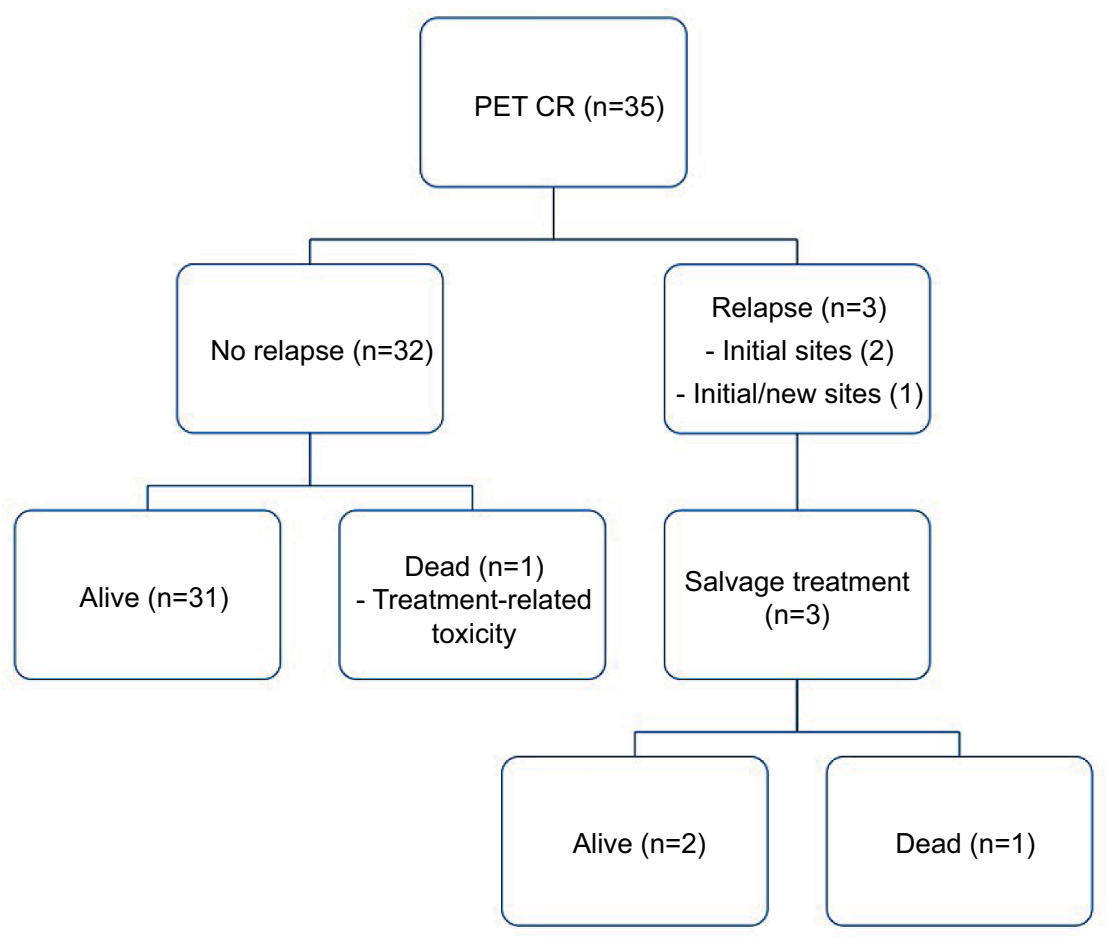

Figure I Outcomes for patients in a CR at the end of chemotherapy.

Abbreviations: CR, complete response; PET, positron emission tomography.

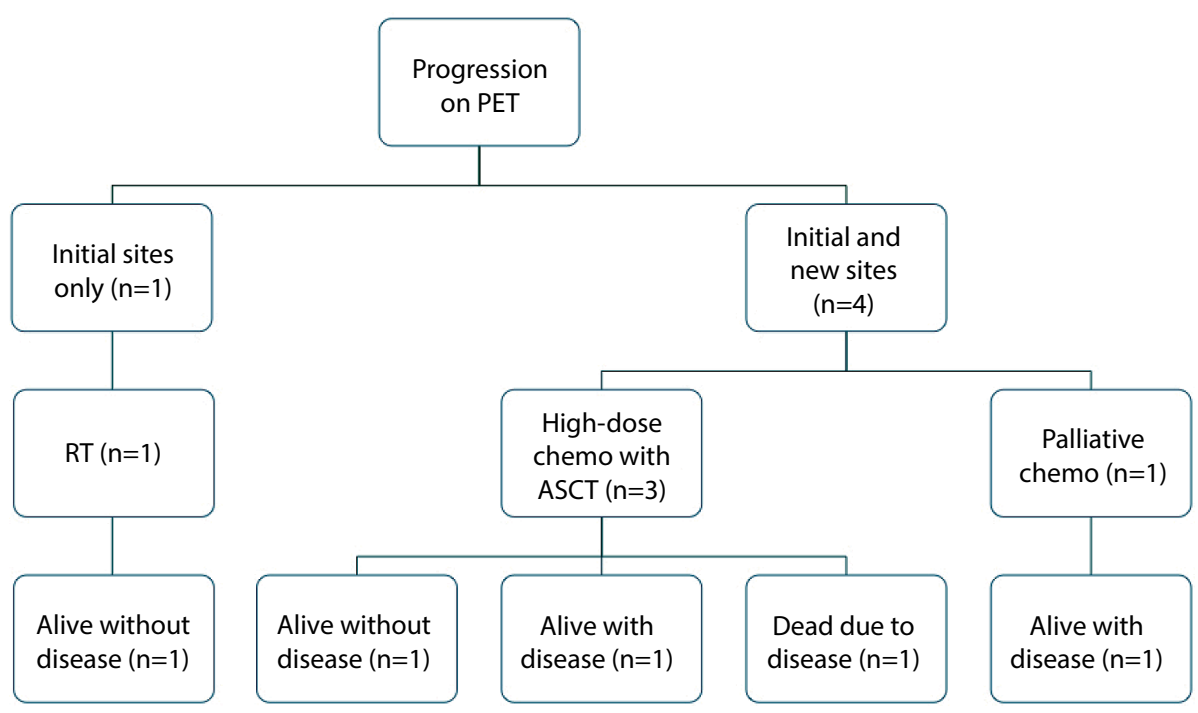

Figure 2 Outcomes for patients with progression on post-chemotherapy PET scan.

Abbreviations: PET, positron emission tomography; RT, radiotherapy; ASCT, autologous stem cell transplant.

\section{Prognostic factors}

Age, bulky disease, stage and the presence of B symptoms were not significant prognostic factors for DFS, PFS or OS; however, patient numbers were small.

\section{Outcomes following consolidative RT}

Five patients had bulky disease at diagnosis, four with bulky mediastinal disease and one patient with bulky left neck disease. Consolidative RT (30 Gy in 15 fractions) was given to four of these five patients. The patient who did not receive consolidative RT was a 25 -year-old female with a bulky mediastinal mass, with a subsequent PET CR after completion of ABVD. Of the four irradiated patients, three patients had a PET CR after ABVD, while one patient had a PET PR prior to the planned consolidative RT. None of the irradiated patients developed a relapse. One patient who had 


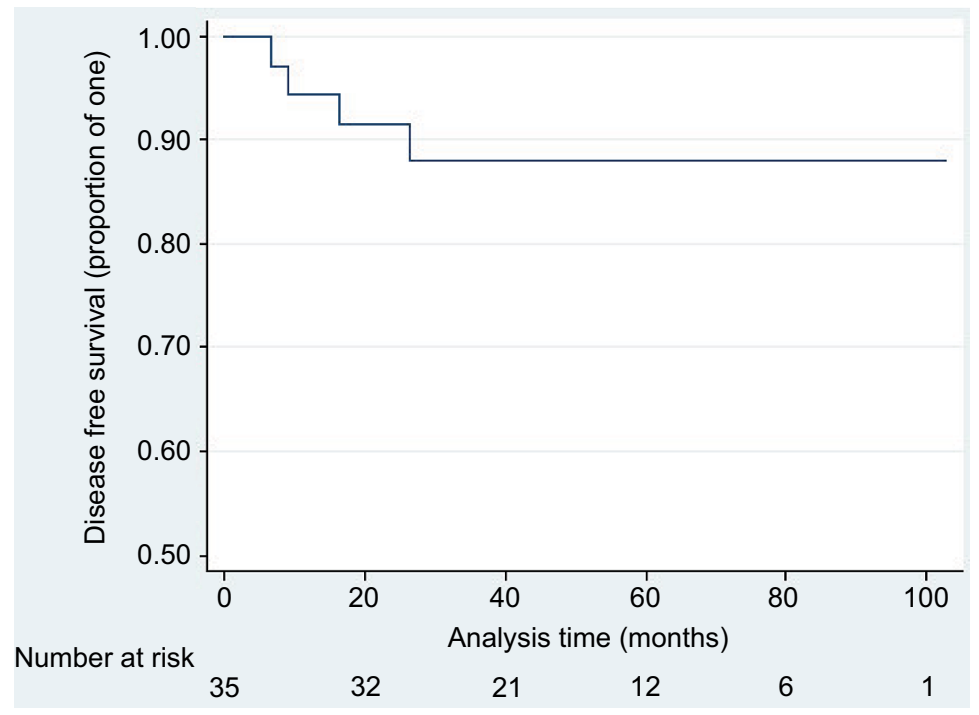

Figure 3 Five-year DFS.

Abbreviation: DFS, disease-free survival.

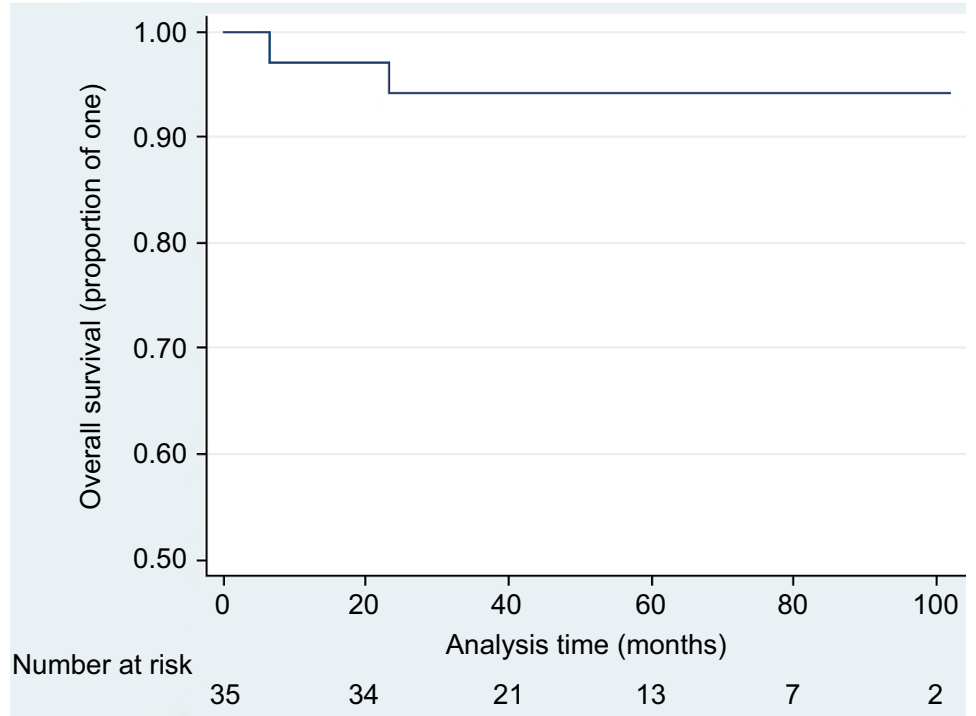

Figure 4 Five-year OS for patients with a PET complete response at the end of chemotherapy. Abbreviations: OS, overall survival; PET, positron emission tomography.

non-bulky disease at diagnosis had a PR in the mediastinum following chemotherapy and commenced consolidative RT. This was ceased after eight fractions after the patient developed symptomatic progression in previously uninvolved sites. Two patients with progressive disease on post-chemotherapy PET received RT (36 Gy in 18 fractions) as part of salvage therapy and both remained disease free at the last follow-up.

\section{Discussion}

The mainstay of treatment for advanced stage HL is chemotherapy, with various regimens found to induce a complete remission. ${ }^{13} \mathrm{ABVD}$ chemotherapy is the most commonly used regimen in our center as it is well tolerated by patients and is significantly less toxic compared with other chemotherapy regimens. ${ }^{6}, 13$ This study demonstrated that patients who achieve a PET CR following ABVD chemotherapy have a low incidence of relapse and an excellent 5-year OS of 94\%.

Among those patients who relapsed following either a CR or PR on PET, most failed at the initial sites of disease, which is consistent with other patterns of relapse studies, although these studies used different chemotherapy regimens. ${ }^{9}{ }^{10}$ Young et al demonstrated that $92 \%$ of patients with relapse following combination chemotherapy with mechlorethamine, vincristine, procarbazine and prednisone had some involvement in 
an initial site of disease, most commonly nodal relapses. ${ }^{10}$ Similarly, Yahalom et al found that $86 \%$ of relapses were at sites of original involvement and most patients had relapses in nodal areas $(85 \%){ }^{9}$

Unfortunately, due to the low number of patients and the low relapse events, the prognostic factors to define patient groups at a higher risk of relapse who may benefit from consolidative RT could not be determined. Traditionally, consolidative RT is given to patients with bulky disease at diagnosis or residual disease on imaging following chemotherapy, in order to minimize the risk of relapse. ${ }^{12,13}$ In the current study, four patients completed consolidative RT for initial bulky disease. Three patients had a PET CR following ABVD and one had a PET PR. None have had relapses, demonstrating excellent disease control following consolidative RT.

The use of RT routinely in this patient population is controversial due to the long-term RT toxicities of second malignancies and cardiovascular disease. ${ }^{6,12}$ Currently available data on the use of RT are varied in results. A Phase III study by Aleman et al suggested RT can be safely omitted (and, therefore, RT toxicities avoided) in patients with a CR after hybrid chemotherapy. ${ }^{18}$ However, the chemotherapy regimen described in this study is no longer used due to significant toxicity. Most patients with bulky disease did not achieve a CR. Of the 247 patients with a PR, 227 received consolidation RT and had a similar event-free survival and OS rates as patients with a $\mathrm{CR},{ }^{21}$ suggesting a benefit for RT in patients with a PR following chemotherapy. A randomized study by Laskar et $\mathrm{al}^{15}$ reported a benefit with the use of RT following ABVD in certain subgroups, including advanced stage, bulky disease and B symptoms, although half the patients in this study were under 15 years of age. Multiple nonrandomized studies have also shown better outcomes following consolidation RT in advanced HL. ${ }^{16,17}$ However, all of these studies used conventional CT scans for posttreatment response assessment.

The use of PET/CT for end of treatment response assessment is more accurate than CT alone in HL and has been part of standard care at our center since $2005 .{ }^{11}$ In the German HD15 study, Engert et $\mathrm{al}^{22}$ used PET response to guide RT in advanced HL treated with BEACOPP. RT was only given to patients with PET-avid residual masses 2.5 cm or larger after BEACOPP. Patients with PET-negative residual masses were found to have a similar PFS to those with a CR based on conventional CT (4-year PFS 92.6\% vs $92.1 \%$ ), whereas patients with PET-positive residual masses had a lower 4-year PFS (86.2\%). There is limited data on post-chemotherapy PET to guide the use of RT following ABVD chemotherapy. ${ }^{12}$ In the current study, two patients with PET-positive residual masses following ABVD received consolidative RT. One patient had initial bulky disease and was already planned for RT and remains relapse free. The other patient had residual PET avidity in an initial site of disease (which was non-bulky) and progressed in new sites during consolidative RT.

An interim PET scan is increasingly being used by clinicians to ensure response to therapy and to identify progression, in order to escalate treatment early. ${ }^{11}$ Interim PET scans changed management in $17 \%$ of the current study patients. However, because interim PET scans were performed in only $70 \%$ of the study patients and were performed after a range of cycles of $A B V D$, the role of interim PET scan to adapt to treatment in advanced HL has not been examined in this study. More information will become available to address this issue with the long-term results of recently closed or currently recruiting prospective trials examining chemotherapy intensification based on PET response after two cycles (PET2) of chemotherapy. These include studies by Cancer Research UK (Response Adjusted Therapy for Hodgkin Lymphoma, NCT00678327), ${ }^{23}$ a USA intergroup (S0816, NCT00822120) ${ }^{24}$ and an Italian lymphoma group (GITIL HD0607, NCT00795613). ${ }^{25}$ In these studies, patients are given two cycles of ABVD followed by a PET scan. If they are found to have PET-positive disease after two cycles, chemotherapy is escalated. If they have PET-negative disease, they continue with another four cycles of ABVD, or in the Response Adjusted Therapy for Hodgkin Lymphoma study, patients are randomized to ABVD or AVD (doxorubicin, bleomycin, dacarbazine).

The role of RT based on PET response is being examined by several groups including two Italian studies (HD0607, NCT00795613 and HD0801, NCT00784537) in which patients treated with six cycles of ABVD with PET2-negative disease are randomized to either adjuvant RT or no RT to bulky sites. ${ }^{25,26}$ Until the long-term data from these prospective studies are published, changing treatment based on the interim PET/CT is not recommended unless disease progression is found. ${ }^{11}$

Variations in the interpretation of response on PET scans lead to the development of the five-point scale Deauville score, ${ }^{27}$ a reproducible scoring system, to report treatment response. ${ }^{11}$ Most patients in the current study were treated prior to the introduction of the Deauville criteria, and therefore, they were not adopted for response assessment. However, the Deauville criteria are now recommended as 
the favored reporting method and have been increasingly incorporated into practice. ${ }^{11}$

\section{Limitations}

Limitations of this study include the retrospective nature, the small patient numbers and the short follow-up period, thereby restricting the conclusions that can be drawn. However, the study suggests that patients with a PET CR following ABVD chemotherapy have a low overall relapse rate and an excellent 5-year OS. Patient selection for consolidative RT remains ill-defined and factors such as the patient's age, sex, site of RT and PET response must be considered.

\section{Conclusion}

This study demonstrated excellent OS in patients with advanced stage HL who achieve a PET CR following ABVD chemotherapy. Relapse rates were low and occurred predominantly at the initial sites of disease. Patients treated with consolidative RT either for bulky disease or due to an incomplete response on PET after chemotherapy had excellent local control with no in-field relapses observed. However, predictors for recurrence were not identified in this study due to the small patient numbers and events. We await the results of ongoing randomized studies which use risk-adapted strategies, for further clarification of the appropriate patient group requiring consolidative $\mathrm{RT}$.

\section{Acknowledgments}

The abstract of this paper was presented at the International Congress on Malignant Lymphoma (ICML) Lugano, Switzerland 2015 and the European Society for therapeutic Radiation Oncology Annual Meeting (ESTRO) Barcelona, Spain 2015 as an abstract presentation with interim findings. The poster's abstract was published in "Poster Abstracts" in Hematological Oncology, Volume 33, Issue Supplement S1, Pages 1-365 and Radiotherapy and Oncology, April 2015, Volume 115, Supplement 1, S1-S997.

\section{Disclosure}

The authors report no conflicts of interest in this work.

\section{References}

1. Canellos GP, Anderson JR, Propert KJ, et al. Chemotherapy of advanced Hodgkin's disease with MOPP, ABVD, or MOPP alternating with ABVD. N Engl J Med. 1992;327(21):1478-1484.

2. Duggan DB, Petroni GR, Johnson JL, et al. Randomized comparison of $\mathrm{ABVD}$ and MOPP/ABV hybrid for the treatment of advanced Hodgkin;s disease: report of an intergroup trial. J Clin Oncol. 2003;21(4):607-614.
3. Federico M, Luminari S, Iannitto E, et al. ABVD compared with BEACOPP compared with CEC for the initial treatment of patients with advanced Hodgkin's lymphoma: results from the HD2000 Gruppo Italiano per lo Studio dei Linfomi Trial. J Clin Oncol. 2009;27(5):805-811.

4. Gordon LI, Hong F, Fisher RI, et al. Randomized phase III trial of ABVD versus Stanford $V$ with or without radiation therapy in locally extensive and advanced-stage Hodgkin lymphoma: an intergroup study coordinated by the Eastern Cooperative Oncology Group (E2496). J Clin Oncol. 2013;31(6):684-691.

5. Hoskin PJ, Lowry L, Horwich A, et al. Randomized comparison of the stanford $\mathrm{V}$ regimen and ABVD in the treatment of advanced Hodgkin's Lymphoma: United Kingdom National Cancer Research Institute Lymphoma Group Study ISRCTN 64141244. J Clin Oncol. 2009;27(32):5390-5396.

6. Johnson PW, Radford JA, Cullen MH, et al. Comparison of ABVD and alternating or hybrid multidrug regimens for the treatment of advanced Hodgkin's lymphoma: results of the United Kingdom Lymphoma Group LY09 Trial (ISRCTN97144519). J Clin Oncol. 2005;23(36): 9208-9218.

7. Santoro A, Bonadonna G, Valagussa $P$, et al. Long-term results of combined chemotherapy-radiotherapy approach in Hodgkin's disease: superiority of ABVD plus radiotherapy versus MOPP plus radiotherapy. J Clin Oncol. 1987;5(1):27-37.

8. Skoetz N, Trelle S, Rancea M, et al. Effect of initial treatment strategy on survival of patients with advanced-stage Hodgkin's lymphoma: a systematic review and network meta-analysis. Lancet Oncol. 2013;14(10):943-952.

9. Yahalom J, Ryu J, Straus DJ, et al. Impact of adjuvant radiation on the patterns and rate of relapse in advanced-stage Hodgkin's disease treated with alternating chemotherapy combinations. J Clin Oncol. 1991;9(12):2193-2201.

10. Young RC, Canellos GP, Chabner BA, Hubbard SM, DeVita VT Jr. Patterns of relapse in advanced Hodgkin's disease treated with combination chemotherapy. Cancer. 1978;42(2 Suppl):1001-1007.

11. Barrington SF, Mikhaeel NG, Kostakoglu L, et al. Role of imaging in the staging and response assessment of lymphoma: consensus of the International Conference on Malignant Lymphomas Imaging Working Group. J Clin Oncol. 2014;32(27):3048-3058.

12. Johnson P, McKenzie H. How I treat advanced classical Hodgkin lymphoma. Blood. 2015;125(11):1717-1723.

13. Moccia AA, Donaldson J, Chhanabhai M, et al. International prognostic score in advanced-stage Hodgkin's lymphoma: altered utility in the modern era. J Clin Oncol. 2012;30(27):3383-3388.

14. Cheson BD, Fisher RI, Barrington SF, et al. Recommendations for initial evaluation, staging, and response assessment of Hodgkin and non-Hodgkin lymphoma: the Lugano classification. J Clin Oncol. 2014;32(27):3059-3068.

15. Laskar S, Gupta T, Vimal S, et al. Consolidation radiation after complete remission in Hodgkin's disease following six cycles of doxorubicin, bleomycin, vinblastine, and dacarbazine chemotherapy: is there a need? J Clin Oncol. 2004;22(1):62-68.

16. Johnson PW, Sydes MR, Hancock BW, Cullen M, Radford JA, Stenning SP. Consolidation radiotherapy in patients with advanced Hodgkin's lymphoma: survival data from the UKLG LY09 randomized controlled trial (ISRCTN97144519). J Clin Oncol. 2010;28(20): 3352-3359.

17. Phan J, Mazloom A, Abboud M, et al. Consolidative radiation therapy for stage III Hodgkin lymphoma in patients who achieve complete response after ABVD chemotherapy. Am J Clin Oncol. 2011;34(5): 499-505.

18. Aleman BM, Raemaekers JM, Tirelli U, et al. Involved-field radiotherapy for advanced Hodgkin's lymphoma. N Engl J Med. 2003;348(24): 2396-2406

19. Hasenclever D, Diehl V. A prognostic score for advanced Hodgkin's disease. International prognostic factors project on advanced Hodgkin's disease. N Engl J Med. 1998;339(21):1506-1514. 
20. Cheson BD, Pfistner B, Juweid ME, et al; International Harmonization Project on Lymphoma. Revised response criteria for malignant lymphoma. J Clin Oncol. 2007;25(5):579-586.

21. Aleman BM, Raemaekers JM, Tomisic R, et al; European Organization for Research and Treatment of Cancer (EORTC) Lymphoma Group. Involved-field radiotherapy for patients in partial remission after chemotherapy for advanced Hodgkin's lymphoma. Int J Radiat Oncol. 2007;67(1):19-30.

22. Engert A, Haverkamp H, Kobe C, et al. Reduced-intensity chemotherapy and PET-guided radiotherapy in patients with advanced stage Hodgkin's lymphoma (HD15 trial): a randomised, open-label, phase 3 non-inferiority trial. Lancet. 2012;379(9828):1791-1799.

23. Clinicaltrials.gov. Fludeoxyglucose F 18-PET/CT Imaging in Assessing Response to Chemotherapy in Patients With Newly Diagnosed Stage II, Stage III, or Stage IV Hodgkin Lymphoma. Available from: https:/clinicaltrials.gov/ct2/show/NCT00678327. Accessed August 31, 2015.
24. Clinicaltrials.gov. S0816 Fludeoxyglucose F 18-PET/CT Imaging and Combination Chemotherapy With or Without Additional Chemotherapy and G-CSF in Treating Patients With Stage III or Stage IV Hodgkin Lymphoma. Available from: https://clinicaltrials.gov/ct $2 /$ show/ NCT00822120. Accessed August 31, 2015.

25. Clinicaltrials.gov. Positron Emission Tomography (PET)-Adapted Chemotherapy In Advanced Hodgkin Lymphoma (HL) (HD0607). Available from: https:/clinicaltrials.gov/ct2/show/NCT00795613. Accessed August 31, 2015.

26. Clinicaltrials.gov. High-dose Chemotherapy and Stem Cell Transplantation, in Patients PET-2 Positive, After 2 Courses of ABVD and Comparison of RT Versus no RT in PET-2 Negative Patients (HD0801). Available from: https://clinicaltrials.gov/ct2/show/NCT00784537. Accessed August 31, 2015.

27. Meignan M, Gallamini A, Haioun C. Report on the first International workshop on Interim-PET-Scan in Lymphoma. Leuk Lymphoma. 2009;50(8):1257-1260.
Blood and Lymphatic Cancer: Targets and Therapy

\section{Publish your work in this journal}

Blood and Lymphatic Cancer: Targets and Therapy is an international, peer-reviewed, open access journal focusing on blood and lymphatic cancer research, identification of therapeutic targets and the optimal use of preventative and integrated treatment interventions to achieve improved outcomes, enhanced survival and quality of life for the
Dovepress

cancer patient. The manuscript management system is completely online and includes a very quick and fair peer-review system. Visit http://www.dovepress.com/testimonials.php to read real quotes from published authors.

Submit your manuscript here: https://www.dovepress.com/blood-and-lymphatic-cancer-targets-and-therapy-journal 